\title{
2.4 Karin Knorr Cetina: Postsozialität
}

\author{
SABINE MAASEN/MARIO KAISER
}

Alt, ja platonisch ist die Idee, wonach uns Theorien helfen, die Welt zu deuten, zu erklären, zu verstehen. Das gilt auch für die Theorien und Konzepte der Soziologie, mit denen wir uns einen Reim auf die Gesellschaft machen - neuerdings heißt sie: Wissensgesellschaft. Während dieses Konzept noch eine spontane Plausibilität erzeugt, erweisen sich andere Begriffe als sperriger. In diesem Fall hilft uns die Gesellschaft, die Soziologie zu verstehen. So klären uns heute Fernsehserien darüber auf, was mit Postsozialität gemeint sein könnte, etwa dank der us-amerikanischen Krimiserie CSI: Den Tätern auf der Spur (Zuiker 2000), die uns seit einer Dekade mit dem Gedanken an eine objektzentrierte Sozialität vertraut macht.

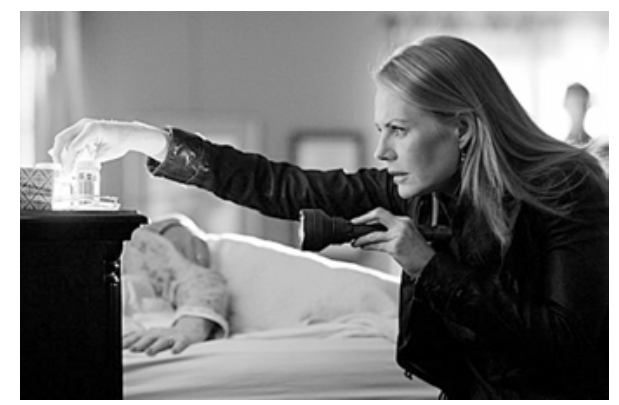

Abbildung 1: Szene aus CSI

Im Gegensatz zu der deutschen Tatort-Reihe, in der Ermittler ihren Weg durch spezifische soziale Milieus von deutschen Städten erfragen müssen, sich dabei häufig von diesen Subkulturen zumindest im Sinne eines 
vorübergehenden going native gefangen nehmen lassen, geschieht in CSI nichts dergleichen. Im Gegenteil. Der Erfolg des Teams, das aus Chemielaboranten, Materialanalysten und Medizinern besteht, beruht gerade darauf, dass es zu den sozialen und kulturellen Umständen des Verbrechens eine fast hyperszientifische Distanz hält. Mehr noch: Die Protagonisten interagieren mit ihren potentiellen Tätern bis zum Schluss der Untersuchungen kaum je direkt, dafür umso vermittelter über Objekte, Dinge, Spuren, Einschusslöcher, DNA-Proben, kleinste Textilrückstände. Das betrifft überdies die Interaktionen im Team selbst. Denn auch diese sind im Gegensatz zu den Beziehungen zwischen den deutschen Tatortermittlern jeglicher psychologischer und sozialer Ambivalenzen entkleidet - zumindest von Ambivalenzen, die über die jeweilige Fach- oder eher: Objektkompetenz hinausgehen. CSI kommt damit einschlägigen Filmen gleich, in denen der obszöne Blick auf das Objekt ausgiebig inszeniert wird - wobei es sich hier um Objekte handelt, die unablässig befragt, untersucht, verwandelt, destilliert, filtriert und fraktioniert werden, bis sie endlich ihre Wahrheiten preisgeben (vgl. Abbildung 1).

Die entscheidende Frage nun lautet: Sind diese Spuren und Objekte lediglich Derivate, Supplemente oder Fetische für das Soziale oder sind sie das Soziale selbst? Folgt man dem Konzept einer Postsozialität, wie es von Karin Knorr Cetina (1997; 2001; 2007a; 2007b) in einer Reihe von Veröffentlichungen umrissen wurde, unterhalten wir in der so genannten Wissensgesellschaft in der Tat: eine Sozialität mit Objekten. Im Klartext bedeutet dass, dass die Objekte selbst zu relevanten Bestandteilen, Mitgliedern oder gar Akteuren der Gesellschaft werden und so ihre alten soziologischen und anthropologischen Bestimmungen hinter sich lassen, denen zufolge Objekte nicht anderes sind als Instrumente oder Waren einer im weitesten Sinne >humanistischen< Sozialität. Die in CSI gesammelten und untersuchten Dinge wären folglich eben nicht nur Spuren von Tätern, sondern bis zu einem gewissen Grade diese Täter selbst. Folgt man der Diagnose einer postsozialen, posthumanistischen Gesellschaft, gilt es daher,

- erstens die »massive Ausbreitung von Objektwelten innerhalb des Sozialen« zur Kenntnis zu nehmen,

- zweitens das soziale Umfeld neu zu bestimmen, »in dem Objekte an die Stelle von Menschen als Interaktionspartner treten und traditionelle, über Interaktion vermittelte soziale Einbettungen ersetzen « und

- drittens dem Umstand Rechnung zu tragen, wonach »zwischenmenschliche Verhältnisse über Objekte vermittelt und diese in zu- 
nehmenden Masse auf Objekte angewiesen sind (Knorr Cetina 2007a: 25).

Heißt eine der Grundfragen der Soziologie, das Verhältnis zwischen Individuum und Gesellschaft zu ergründen, animieren Serien wie CSI da$\mathrm{zu}$, nicht nur die Beziehung selbst, sondern mit ihr auch die entsprechenden Relationsglieder zu überdenken. Denn zwischen Individuum und Gesellschaft haben sich laut Knorr Cetina epistemisch und sozial anspruchsvolle Objekte eingestellt, die beide Seiten in ihrem Wesen nicht unberührt lassen. Vor diesem Hintergrund fordert das Konzept der Postsozialität dazu auf, zum einen die Sozialität von und mit Objekten neu zu bestimmen, zum anderen, Brücken zwischen mehreren, bislang eher isolierten Fachdiskussionen in der Soziologie zu schlagen.

\section{Sozialität mit Objekten}

Karin Knorr Cetina wagt sich mit ihrer These von der "postsozialen Wissensgesellschaft« auf ein Terrain, das bereits von einigen etablierten Konzepten bestellt ist - u.a. Individualisierung, Expertisierung und neue Formen der Wissensproduktion. Sie erweitert diese Landschaft nun um den Aspekt der Technisierung der Wissensgesellschaft und verleiht ihm einen neuen Namen: Postsozialität. Die Grundidee lautet: Die Objektbeziehungen nehmen zu, objektzentrierte Umwelten vermitteln neue Beziehungen und schaffen neue Bindungen für das postsoziale Selbst. Dies geht mit der Expansion gemischter Beziehungen von Subjekte und Objekte her. Knorr Cetina spricht hier von einer »Kreolisierung « (Knorr Cetina 1997: 6) der modernen Gesellschaft.

Postsozialität versteht sich in diesem Sinne als Beschreibung eines Wandlungsprozesses von »epischem Charakter« (Knorr Cetina 2007b: 295). In enger Nachbarschaft zu Diagnosen, die den Wandel vom industriellen zu post-industriellem Leben, von Nationalstaaten zu Weltgesellschaften oder von Moderne zu Postmoderne identifizieren, fokussiert dieses Konzept auf das Soziale und die Bedingungen von Gesellschaftlichkeit. Dies geschieht in dezidierter Entgegensetzung zu Arbeiten, die zwar die »konsumistische Neigung in westlichen Demokratien« (Ritzer 2010) oder das Schwinden der gegenwärtigen menschlichen, ja humanistischen Beziehungen (Bauman 1994; 2009) feststellen, zugleich aber ignorieren, dass dies Hinweise auf, wenn nicht gar Indikatoren für neue Formen von Sozialität sind.

Es ist vor allem die eklatante Technisierung der modernen Wissensgesellschaft, die nicht etwa einer >De-Sozialisierung < gleichkommt, son- 
dern mit einer Expansion von Elementen und Praktiken einhergeht, die bislang nicht als >sozial< bezeichnet wurden. Gleich, ob es um technische Dinge, Konsumgüter, Tauschinstrumente (z.B. Aktien) oder Expertensysteme geht - alle diese Elemente und die mit ihnen verbundenen Praktiken weiten derzeit die sozialen Formen und damit: die soziale Welt aus.

Das Konzept der Postsozialität impliziert einerseits einen spezifischen Objektbegriff, andererseits stützt es sich auf eine veränderte Struktur des Selbst. Was die postsozialen Objekte und ihre zunehmende Präsenz in der sozialen Welt betrifft, so löst Knorr Cetina sich von der Vorstellung material fixierter Dinge und charakterisiert sie demgegenüber als notorisch unbestimmt, sich wandelnd und sukzessive entfaltend. In Anlehnung an Rheinbergers Konzept des epistemischen Dinges (2006) geschieht dies, da die Objekte wie etwa Softwareprogramme in ihre Umwelt eingreifen, mit ihr interagieren, und so quasi-experimentell immer neue Ausgestaltungs- und Verwendungsweisen erfahren - oder durch eine neue Version ersetzt werden müssen. Das Ding ist dementsprechend nie >fertig $<$. Wenn Rheinberger für sein Konzept des epistemischen Dings Derridas Begriff der Différance (Derrida 2004) aufgegriffen hat, geschah dies, um zu zeigen, dass Dinge immer aufs Neue befragt, analysiert, erprobt und in neue Verwendungsmöglichkeiten eingebettet werden müssen. Im Alltag sind es etwa Computerprogramme, die diesen Status einnehmen: Sie sind nie ganz fertig, die nächsten Applikationen, das nächste update ist immer schon in Sicht. Ihre je mögliche Verwendung erschließt sich, indem sie vom Subjekt angeeignet, personalisiert, gelegentlich auch affektiv besetzt werden. Postsoziale Objekte kennzeichnet folglich eine duale Struktur: Sie dienen einerseits dem sofortigen Gebrauch und stellen andererseits für den Nutzer ein unerschöpfliches Entdeckungsprogramm dar, das ein relationales Engagement des Subjekts mit dem Objekt verlangt (Knorr Cetina 2007a: 34).

Genauso wie postsoziale Objekte immer vor ihrer Realisierung stehen, so steht auch das postsoziale Subjekt stets vor seiner Subjektwerdung. Was das Konzept des postsozialen Selbst betrifft, so löst es sich vom »I-you-me«-Modell (Peirce, Mead, Freud), das ein Realität schaffendes $>\mathrm{I}<$ sowie ein kritisches, die Standards der Gesellschaft repräsentierendes $>$ me $<$ (Mead) oder $>$ you $<$ (Peirce) in ein regulatives Verhältnis setzt. Stattdessen orientiert sich der der Begriff eines postsozialen Subjekts an Lacan und fasst »es als eine Struktur von Wünschen zu einem kontinuierlich erzeugten Mangel auf« (Knorr Cetina 2007a: 29f.). Das You repräsentiert nun das idealisierte Selbst im Spiegel oder den perfekten Anderen. Es konstituiert den steten Mangel im Subjekt und damit sein Begehren, das Gewünschte zu erreichen. In technisierten Wissens- 
gesellschaften wird dieser Spiegel indes stets erneuert und modifiziert er institutionalisiert sich in den Medien, in der Werbung, aber auch in Konsumtempeln (Ritzer 1999). Das Subjekt, das ein relationales Engagement mit einem Objekt entwickelt hat, wird durch deren Weiterentwicklung zur Ausbildung immer weiterer Bedürfnisse verlockt (Knorr 2007: 34).

An der Schnittstelle von wünschendem Selbst und veränderbaren Objekten stellen sich postsoziale Relationen ein. Sie bauen auf einer strukturellen Affinität auf, die Subjekte und Objekte - hier per Wunschstruktur, dort per permanenter Re-Definition - in ein wechselseitig konstitutives Verhältnis setzt: Immer neue Begehrensketten provozieren die Herstellung von Objektvariationen (Autos, Computer, Mode) - und vice versa. Postsoziale, insbesondere kommunikationstechnologisch hergestellte Relationen sind signalarm, und erfordern eben deshalb verstärktes interpretatives Engagement. Eben dies sorgt für eine »neue Lässigkeit« gegenüber Computern (Turkle 1998), zu denen Subjekte in vielerlei Beziehungen treten (z.B. informative, beratende, unterhaltende), und wofür das Internet reichhaltiges Anschauungsmaterial liefert.

Hiermit wird eine interobjektive Sozialität beschrieben, die zuweilen auch durch eine Solidarität mit den Objekten hergestellt wird. Die moralische Dimension dieser Sozialität (»Every time I walk on grass I feel sorry because I know the grass is screaming at me«, Mc Clintock) entsteht jedoch nicht aus Höflichkeit oder Sentimentalität, sondern ebenfalls aus Wissen - und zwar (hier:) aus dem Wissen über Pflanzen und ihren Mechanismen der Reaktion auf ihre Umwelt (Knorr Cetina 2007b: 281).

Daraus ergibt sich mit dem Konzept der Postsozialität ein wichtiger Aspekt für das Konzept der Wissensgesellschaft: »Wissensgesellschaften sind nicht einfach Gesellschaften mit mehr Experten, mehr technologischen Infra- und Informationsstrukturen. [...] [Es geht darum,] dass Wissenskulturen ihre Fäden in soziale Prozesse einschießen, den gesamten Komplex der Erfahrungen und Beziehungen, die sich mit der Artikulation von Wissen entfalten und etablieren« (Knorr Cetina 1998: 93). Auch wenn Knorr Cetina der postsozialen Gesellschaft eine >liminale Sozialität< bescheinigt, so geht es ihr keineswegs um eine Verlustanzeige im Sinne eines Schwindens des Sozialen, sondern um die Charakterisierung einer bisher wenig beachteten Dimension der Wissensgesellschaft - um die Transformation des Sozialen.

»Die postsozialen Formen sind nicht reich an Sozialität im alten Sinn, aber sie können in anderer Hinsicht reich sein, und die soziologische Herausforderung 
besteht dann darin, diese neuen Konstellationen sowohl theoretisch als auch empirisch zu analysieren« (Knorr Cetina 2007a: 38).

In diesem Sinne ist Postsozialität als ein Suchbegriff zu verstehen, der rezente Veränderungen des Sozialen, die mit der Entwicklung zur Wissensgesellschaft einhergehen, aufgreift, bündelt und in einen, wenn auch vorläufigen, konzeptionellen Zusammenhang eines »Umrisses« (Knorr Cetina 2007a) stellt: postsoziale Selbste, postsoziale Objekte, postsoziale Beziehungen. Dies geschieht in enger Nachbarschaft zu Entwürfen etwa des »Neosozialen« (Lessenich) oder des »Technosozialen« (ActorNetwork-Theory), Formen der »Hybridisierung « (Latour) und das Selbst als »Cyborg « (Haraway). Sie alle gehen der Beobachtung nach, dass Objekte, also Wissen, Technik und Artefakte, konstitutiver Teil des Gesellschaftlichen sind. In diesem Zusammenhang interessieren sie sich vor allem für die Formen und Folgen von zunehmender Individualisierung und Objektualisierung in der technisierten Wissensgesellschaft

\section{Individualisierung und Objektualisierung}

Objekte im Allgemeinen, wissenschaftliche Gegenstände im Besonderen, bevölkern die Soziologie schon seit geraumer Zeit. Zumeist aber wurde ihnen höchstens ein defizienter oder supplementärer, gleichsam ein Ersatzstatus für das eigentlich Soziale zuerkannt. Mit Ausnahme der jüngeren Wissenschaftssoziologie (Science and Technology Studies) bekam es die Soziologie folglich nur selten mit things as such, vielmehr mit Objekten als Instrumenten oder mit Dingen als Produkten eines sozialen Umwandlungsprozesses zu tun.

So geht das neomarxistische Theorem der Reifizierung oder Verdinglichung (Lukács 1986[1923]) im Anschluss an Marx' Warenfetisch gerade davon aus, dass der moderne Kapitalismus ehemals soziale Beziehungen und Tatsachen wie Arbeitsverhältnisse in immer stärkerem Ausmaße in Dinge, d.h. Waren verwandelt, die auf diese Weise eine Objektivität oder Naturalität erlangen. »Verdinglichung bedeutet«, so schreiben noch Berger und Luckmann in den 1960er Jahren, »menschliche Phänomene aufzufassen, als ob sie Dinge wären, das heißt als außeroder gar übermenschlich. [...] Verdinglichung ist die Auffassung von menschlichen Produkten, als wären sie etwas anderes als menschliche Produkte« (Berger/Luckmann 1980[1966]: 94f.). Entsprechend der Verdinglichungsthese werden wir zwar in einem gigantischem Ausmaß von Dingen bzw. Waren heimgesucht, doch diese sind eigentlich nicht sie selbst - sie sind immer schon die zweitrangigen Als-Ob-Produkte eines 
kapitalistischen Transformationsprozesses, in dem ursprünglich menschliche und soziale Phänomene zu Dingen gemacht werden.

Ebenfalls auf zweite Plätze verwiesen werden Dinge, wenn sie als Mittel oder Instrumente im Rahmen einer technischen oder »instrumentellen Vernunft « (Horkheimer und Adorno 1981; Habermas 1969) konzipiert werden. Im Vergleich zu der sozialtheoretisch bevorzugten Thematisierung von Zwecken und Zielen verschwinden die >mittelbaren Dinge hinter diesem Horizont fast ganz. Sie machen sich gegenüber dem Handeln, das sie als >Zeug< im Sinne Heideggers (2001[1927]: §16) benutzt, nahezu unsichtbar - »aufdringlich« (ebd.) werden sie nur dann, wenn sie den Fluss des instrumentellen Handelns stören und unterbrechen. Kurz: Während das Konzept der Reifikation Objekte stets als etwas anderes, etwa als verdinglichte Arbeit in Blick nimmt, lässt die vernunftkritische Diagnose einer zunehmenden Technisierung und Instrumentalisierung die Objekte qua Instrumente zugunsten der Fokussierung auf Zwecke und Ziele nahezu unbeachtet. Irritation löst höchstens noch die Frage aus, was heutzutage (z.B. menschliche Embryonen) der Instrumentalisierung unterworfen wird.

Die soziologische Deklassierung der Objekte zugunsten des Sozialen erfuhr erst in der jüngeren Wissenschaftssoziologie eine Korrektur. Im Anschluss an die mikrosoziologischen Laborstudien (Knorr Cetina 1981; Latour/Woolgar 1979) erregten zunächst die Widerstände Aufmerksamkeit, die Untersuchungsobjekte der sozialen Praxis auferlegen und diese zu einem >Darumherumarbeiten<zwingen (Pickering 1995). Einsichten wie diese animierten die Wissenschaftssoziologie wie auch die Wissenschaftsgeschichte zu einer produktiven Aneignung philosophischer Konzepte, unter ihnen etwa Bachelards Erkenntnishindernisse (1978) oder Derridas Konzeption einer Materialität von Zeichen (1994a; 1994b). Jenseits einer einseitigen soziologischen Vereinnahmung als bloße Waren oder Instrumente erschlossen sich damit für die Soziologie theoretische Optionen, wissenschaftliche und technische Dinge auf eine Weise $\mathrm{zu}$ thematisieren, dass an ihnen ihr semiotisches oder soziales Eigenleben durchscheinen konnte (Rheinberger 2006; Latour 1999).

Knorr Cetinas Begriff einer Sozialität mit Objekten nimmt diesen Faden auf, trägt sie jedoch aus dem Labor in die Wissensgesellschaft charakteristisch für sie ist, dass sie zunehmend mit epistemisch anspruchsvollen Objekten Wissenkulturen angereichert wird: »society [...] is now more inside knowledge processes than outside« (Knorr Cetina 1997: 8). Und da diese Wissenskulturen sich in erster Linie um Objektwelten formieren, ereignet sich in dieser Gesellschaft eine nie dagewesene Orientierung an Objekten: als Quellen des Selbst, als Quellen von 
Intimität und geteilter Subjektivität sowie als Quellen sozialer Integration (ebd.: 9).

Mit diesem Befund steht Knorr Cetina nicht allein. Auch Bruno Latour hat in seinem Versuch zu einer symmetrischen Anthropologie (2008) wissenschaftliche Objekte und technische Artefakte gleichsam aus dem Labor befreit und über die ganze Gesellschaft verteilt. Ähnlich wie Knorr Cetina, doch radikaler noch räumt auch er den Dingen einen akteursähnlichen Status ein. In Form von Handlungsprogrammen tragen sie in sich eine Handlungsfähigkeit [agency], denen die menschlichen Akteure notgedrungen Folge zu leisten haben - etwa, wenn sie vermeiden wollen, beim Überfahren einer Bodenschwelle zur Tempodrosselung, d.h. eines >schlafenden Gendarmen<, ihr Auto zu beschädigen. Dementsprechend sind es so mundane Objekte wie Sitzgurte oder automatische Türschließer, die laut Latour unsere Gesellschaft in einem MaBe härten, wie es die Sprache bisher noch nicht vermochte: »technology is society made durable« (Latour 2000).

Während Latour allerdings sein Augenmerk gänzlich auf den Aufstieg von Dingen zu so genannten Aktanten richtet und sie als soziologische Phänomene sui generis zu etablieren sucht, blendet er die subjektivitätstheoretischen Implikationen dieser Entwicklung aus. Es ist genau diese Leerstelle, auf die die Diagnose einer Postsozialität eine Antwort zu geben versucht. Denn laut Knorr Cetina ist die zunehmende Objektualisierung, die nicht zu verwechseln ist mit der neomarxistischen Verdinglichung, das unmittelbare Korrelat zum bekannten Befund der Individualisierung.

Wenn im Rahmen von Postsozialität die Rede von Individualisierung ist, betrifft diese nicht so sehr deren erste Welle, wie sie bereits Max Weber oder Emile Durkheim ins Auge fassten, sondern die Entwicklungen nach dem Zweiten Weltkrieg, die etwa durch Ulrich Beck (1986) oder Anthony Giddens (1997) diagnostiziert wurden. Nicht selten wird dieser Prozess in Zusammenhang mit einem Rückzug des Sozialen in Verbindung gebracht, der die Subjekte einerseits aus einer Solidargemeinschaft freisetzt oder - in den Worten von Giddens - entbettet, andererseits sie dazu bringt, riskante Freiheiten (Beck und Beck-Gernsheim 1994) in Kauf zu nehmen. Die zunehmende Betonung der Selbstverantwortung und die damit einher gehenden Risiken sind überdies Thema der gouvermentality studies, die sich im Anschluss an Foucault der Frage angenommen haben, wie die von Subjekten praktizierte Selbststeuerung und -regierung sich mit übergeordneten Regierungstechnologien verzahnen. Und von dieser Seite stammt auch der Vorschlag, das Verhältnis von Individuum und Gesellschaft in der neoliberalen Gouvernementalität als neosozial (Lessenich 2003) zu bezeichnen. »Die Gesell- 
schaft konstitutiert sich als Subjekt, das gemeinwohlkompatibles Handeln der Subjekte einklagt - und das sich gegen jene Individuen schützen und verteidigen muss, deren Verhalten der Gesellschaft Risiken auferlegt. Die Gesellschaft wird zum Bezugspunkt des Sozialen « (Lessenich 2003: 89). Dies geschieht heute vermehrt in wissensbasierten, technosozialen Arrangements präventiver Risikoabwehr (z.B. genetisches Screening, Navigationssysteme).

Dass die gegenwärtige Wissensgesellschaft von einer Veränderung des Sozialen in Gestalt einer Postsozialität geprägt ist, unterschlägt keineswegs, dass der Begriff der Gesellschaft, der noch stark an der Idee des Sozial- und Nationalstaates partizipierte, im Zeitalter der Globalisierung viel von seiner Bedeutung verloren hat (Knorr Cetina 2007a: 28). Doch die im Zuge der Individualisierung vollzogene Entbettung aus einer emphatischen Sozialität, aus als >ursprünglich< gedachten sozialen Beziehungen, wird durch eine verstärkte Objektualisierung beantwortet - eine affektive Bindung von Subjekten an Objekte: »Was postsoziale Theorie anstatt des schlichten Szenarios einer >De-Sozialisierung< anbietet ist eine Analyse neuer, alternativer Formen der Selbst- und Fremdbindung « (ebd. 5). Programmatisch formuliert hätten Analysen dieser Art zur Aufgabe, beides in ihrem Wechselspiel zu berücksichtigen: Eine zunehmende Individualisierung als auch eine komplementäre, möglicherweise auch kompensatorisch operierende Objektualisierung.

\section{Schluss}

Das Konzept der Postsozialität gehört zwar noch nicht zum Standardvokabular in Medien und Wissenschaftspolitik, hat jedoch durch seine Vernetzung mit verwandten Konzepten und Theorien gegenwärtig an Plausibilität gewonnen. Neben Wissenschafts- und Technikforschung interessiert es vor allem in theoretischen und zeitdiagnostischen Debatten. Ein wichtiges gesellschaftstheoretisches Thema kreist um die Beobachtung, dass der heutigen Gesellschaft der Gesellschaftsbegriff abhanden $\mathrm{zu}$ kommen scheint, zumindest »nicht mehr der fraglose Horizont der Gegenwartsdiagnose « (Bude 2002) ist. Auch Karin Knorr Cetina geht mit ihrem Konzept der Postsozialität davon aus, dass nicht mehr die Gesellschaft, sondern das Leben der eigentliche Gegenstand des Räsonnements und der Regulierung sei. Indikativ dafür ist die Weise, in der in der Folge biowissenschaftlicher Innovationen der lebendige Körper heute weniger als organisches Substrat denn als molekulare Software begriffen wird, die >gelesen< und >umgeschrieben< werden kann. Biopolitisch stellt sich nun die Frage nach der Bedeutung von modulierbarem 
Leben innerhalb dieser politisch-technischen Konstellation (z.B. Giorgio Agamben 2007): Die Behauptung ist, dass Leben das zentrale Scharnier der Gestaltung, Veränderung von Individuen und Gesellschaften sei. Individuelles life enhancement, Biopolitik, die Vorrangstellung der life sciences, etc. - all dies indiziert, nicht nur für Knorr Cetina, dass wir gegenwärtig einen Übergang von einer »Kultur des Sozialen« zu einer »naturzentrierten Kultur« beobachten (Knorr Cetina 2007a: 36).

Leben avanciert zugleich zur zentralen gesellschaftstheoretischen Kategorie. Gerade jetzt erscheint es vielen soziologischen Beobachtern wichtig, »eine Soziologie zu erfinden, die das Soziale nicht als Ausgangspunkt, sondern als Resultat einer Koproduktion von Gesellschaft und Natur begreift « (Lemke 2007: 254).

Das Konzept der Postsozialität bietet hierfür eine bedenkenswerte Perspektive, die vielversprechende Analysen auslösen kann. Klar ist noch nicht, ob es sich um eine >Übergangssemantik<, einen Epochenbegriff handelt - Nick Stokes vom CSI-Team ist sich überdies nicht sicher, ob es immer die effektivere Form von Sozialität ist:

Hodges: I Ididn't page you.

Nick Stokes: No. I just figured I'd come by.

Hodges: $\quad$ You're checking up on me again.

Nick Stokes: No, I'm checking up on my evidence.

Hodges: Do you think if you hover the FTIR will work faster?

Nick Stokes: Yes, Hodges, that's what I think.

\section{Literatur}

Agamben, Giorgio (2007): Homo sacer: die souveräne Macht und das nackte Leben. Frankfurt a.M.: Suhrkamp.

Bachelard, Gaston (1978): Die Bildung des wissenschaftlichen Geistes. Beitrag zu einer Psychoanalyse der objektiven Erkenntnis. Frankfurt a.M.: Suhrkamp.

Bauman, Zygmunt (1994): Alone again: ethics after certainty. London: Demos.

Bauman, Zygmunt (2009): Postmoderne Ethik. Hamburg: Hamburger Edition.

Beck, Ulrich (1986): Risikogesellschaft: Auf dem Weg in eine andere Moderne. Frankfurt a.M.: Suhrkamp.

Beck, Ulrich/Beck-Gernsheim, Elisabeth (Hg.) (1994): Riskante Freiheiten: Individualisierung in modernen Gesellschaften. Frankfurt a.M.: Suhrkamp. 
Bude, Heinz (2002): »Die normative Kraft des Physischen. Die Soziologie hat ausgedient. Sie taugt nicht mehr zur Erklärung des Gen- und Computerzeitalters. Fragen nach dem Anfang des Lebens und der Herkunft des Geistes können nur die Biowissenschaften beantworten«. In: Tagesspiegel, Januar 5.

Derrida, Jaques (1994a): Die Schrift und die Differenz. Frankfurt a.M.: Suhrkamp.

Derrida, Jaques (1994b): Grammatologie. Frankfurt a.M.: Suhrkamp.

Derrida, Jaques (2004): »Die différance«. In: Engelmann, Peter (Hg.) Postmoderne und Dekonstruktion. Texte französischer Philosophen der Gegenwart. Stuttgart: Reclam.

Giddens, Anthony (1997): Konsequenzen der Moderne. Frankfurt a.M.: Suhrkamp.

Habermas, Jürgen (1969): Technik und Wissenschaft als >Ideologie< Frankfurt a.M.: Suhrkamp.

Heidegger, Martin (2001): Sein und Zeit. Tübingen: Max Niemeyer.

Horkheimer, Max/Adorno, Theodor W. (1981): Dialektik der Aufklärung: Philosophische Fragmente. Frankfurt a.M.: Suhrkamp.

Knorr Cetina, Karin (1981): The Manufacture of Knowledge: an Essay on the Constructivist and Contextual Nature of Science. Oxford: Pergamon Press.

Knorr Cetina, Karin (1997): »Sociality with Objects: Social Relations in Postsocial Knowledge Societies«. In: Theory, Culture \& Society 14, Nr. 4, S. 1-30. doi:10.1177/026327697014004001.

Knorr Cetina, Karin (2001): »Postsocial Relations: Theorizing Sociality in a Postsocial Environment «. In: Ritzer, George/Smart, Barry (Hg.): Handbook of Social Theory. London; Thousand Oaks, CA: Sage, S. 520-536.

Knorr Cetina, Karin (2007a): »Umrisse einer Soziologie des Postsozialen«. In: Meyer, Lars/Pahl, Hanno (Hg.): Kognitiver Kapitalismus: Soziologische Beiträge zur Theorie der Wissensökonomie. Marburg: Metropolis, S. 25-39.

Knorr Cetina, Karin (2007b): »Postsoziale Beziehungen: Theorie der Gesellschaft in einem postsozialen Kontext«. In: Bonacker, Thorsten/Reckwitz, Andreas (Hg.): Kulturen der Moderne: Soziologische Perspektiven der Gegenwart. Frankfurt a.M.: Campus, S. 267-300.

Knorr Cetina, Karin D. (1998): »Sozialität mit Objekten. Soziale Beziehungen in posttraditionalen Wissensgesellschaften «. In: Rammert, Werner (Hg.): Technik und Sozialtheorie, Frankfurt a.M.: Campus, S. 83-120. 
Latour, Bruno (1999): One More Turn After the Social Turn... In: Biagioli, Mario (Hg.) The Science Studies Reader, New York/London: Routledge, S. 276-289.

Latour, Bruno (2000): Technology is Society Made Durable. In: Grint, Keith (Hg.) Work and Society: A Reader. Malden, MA: Polity Press, S. 1-53.

Latour, Bruno (2008): Wir sind nie modern gewesen: Versuch einer Symmetrischen Anthropologie. 1. Aufl. Suhrkamp Verlag.

Latour, Bruno/Woolgar, Steve (1979): Laboratory Life: The Social Construction of Scientific Facts. Beverly Hills: Sage.

Lemke, Thomas (2007): Die Natur in der Soziologie. Versuch einer Positionsbestimmung. In: Leviathan 35, Nr. 2, S. 248-255.

Lessenich, Stephan (2003): Soziale Subjektivität. In: Mittelweg 36, Nr. 4, S. 80-93.

Lukács, Georg (1986): Die Verdinglichung und das Bewusstsein des Proletariats. In: Geschichte und Klassenbewusstsein. Studien über marxistische Dialektik. Neuwied/Berlin: Luchterhand.

Pickering, Andrew (1995): The mangle of practice: time, agency, and science. Chicago/IL: University of Chicago Press.

Rheinberger, Hans-Jo rg (2006): Experimentalsysteme und epistemische Dinge: eine Geschichte der Proteinsynthese im Reagenzglas. Frankfurt a. M.: Suhrkamp.

Ritzer, George (2010): Enchanting a disenchanted world : continuity and change in the cathedrals of consumption. Los Angeles: SAGE.

Turkle, Sherry (1998): Leben im Netz: Identiät in Zeiten des Internet. Reinbek bei Hamburg: Rowohlt.

Zuiker, Anthony E. (2000): CSI: Crime Scene Investigation. Kriminalserie. CBS. 